'Instituto de Políticas Públicas en Salud, Facultad de Medicina, Universidad San Sebastián, Santiago.

${ }^{2}$ Facultad de Medicina, UDA Ciencias de la Salud, carrera de Fonoaudiología, Pontificia Universidad Católica de Chile. ${ }^{a}$ Cirujano Dentista. bDoctorando en Salud Pública. 'Fonoaudiólogo.

${ }^{\mathrm{d} B e c a r i o ~ p a r a ~ e s t u d i o s ~ d e ~}$ Doctorado en Chile, CONICYTT.

Recibido el 10 de septiembre de 2015, aceptado el 9 de marzo de 2016.

Correspondencia a: María José Monsalves Teléfono: (56 2) 22606566. maria.monsalves@uss.cl

\section{Validación del test Short Assessment of Health Literacy for Spanish- speaking Adults en Chile, para medir alfabetización en salud}

\author{
MARÍA JOSÉ MONSALVES ${ }^{1, a, b, d}$, JAIME MAÑALICH ${ }^{1}$, \\ EDUARDO FUENTES ${ }^{2, \mathrm{~b}, \mathrm{c}, \mathrm{d}}$
}

\section{Validation of the short assessment of health literacy for spanish-speaking adults test in Chile}

Background: Health literacy is defined as the degree to which individuals obtain, process and understand basic health information and services. It is necessary to make appropriate decisions about their health. Evidence has shown that the level of health literacy is critical to the prognosis of chronic diseases. The Short Assessment of Health Literacy for Spanish-speaking Adults (SAHLSA-50) is a short and simple health literacy adult assessment. Aim: To determine the validity and reliability indicators of SAHLSA-50 in Chilean adults. Material and Methods: The survey was applied to 84 older adults living in high and low income neighborhoods. Results: The survey had an adequate construct validity and reliability, its Comparative Fit Index was 0.93, its Tucker-Lewis index was 0.927 and its Root Mean Square Error of Approximation was 0.044. "Close fit" was not statistically significant $(p=0.828)$. Reliability was estimated by K-Richardson, which reported a good outcome (0.9255). Despite the good global indicators obtained, it is necessary to pay attention to some items that would fail to explain the "Health literacy" construct or were beyond the parameters of difficulty and discrimination proposed by the authors of the test. Conclusions: We propose this test as a useful tool to assess health literacy in the adult population in Chile. Its use and incorporation into local research can be especially recommended in the areas of education and health promotion.

(Rev Med Chile 2016; 144: 604-610)

Key words: Health Education; Health Literacy; Health Promotion.
$\mathrm{E}$ 1 concepto de alfabetización en salud hace referencia a los conocimientos y competencias de las personas para satisfacer las complejas demandas relacionadas con su salud en la sociedad actual. Este constructo se introdujo en la década de 1970, teniendo una creciente importancia en las áreas de salud pública y promoción de la salud ${ }^{1}$.

La alfabetización en salud se ha definido como el grado en que los individuos obtienen, procesan y comprenden información básica de salud y servicios; necesaria para tomar decisiones de salud apropiadas. La relevancia de la alfabetización en salud radica en la visión de colocar la propia salud, la de la familia y la comunidad en su contexto. Se dirige a la comprensión de los factores que están influyendo en la propia salud, y saber cómo abordarlos. Una persona con un nivel adecuado de conocimientos sobre salud tiene la capacidad de asumir la responsabilidad de su propia salud, 
así como la salud de su familia y favorecer la salud de su comunidad ${ }^{1-3}$.

La carga de enfermedad del siglo XXI se caracteriza por la elevada prevalencia de las condiciones crónicas no trasmisibles. Para los pacientes y familias afectados, el enfoque clínico clásico, paternalista y dependiente no produce los resultados deseados, porque estas enfermedades requieren el empoderamiento y automanejo de situaciones de cada día, por toda la vida. Varios estudios han demostrado que el nivel de Alfabetización en Salud es crítico para el pronóstico de estas enfermedades ${ }^{4,5}$.

A nivel mundial existen varios instrumentos que permiten medir la alfabetización en salud de las personas ${ }^{6-9}$. La mayor parte de estos instrumentos han sido desarrollados en inglés en contextos diferentes al chileno. Sin embargo, es posible encontrar algunas herramientas que pueden ser utilizados con fines de investigación, clínicos o de planificación de programas en habla hispana. El Short Assessment of Health Literacy for Spanish-speaking Adults (SAHLSA-50) es un instrumento que ha sido diseñado y validado para evaluar la capacidad de leer y entender los términos médicos comunes de un adulto de habla hispana. Este test permite una evaluación rápida y práctica de la alfabetización en salud de personas adultas ${ }^{10}$. En Chile a la fecha no contamos con un instrumento que permita obtener esta medición.

\section{Material y Método}

Se realizó un estudio de corte transversal a un total de 84 adultos mayores de 35 años de las comunas de Providencia y Cerro Navia, con una media de 59 años de edad, en donde $52,4 \%$ es mayor de 60 años. Se aplicó el cuestionario "Short Assessment of Health Literacy for Spanish-speaking Adults" (SAHLSA) con el objetivo de determinar indicadores de validez y confiabilidad del instrumento, posterior a la firma del consentimiento informado. El diseño de estudio se enmarca en un proyecto de investigación de alfabetización en salud realizado por el Instituto de Políticas Públicas en Salud de la Universidad San Sebastián, autorizado por el Comité de ética de la misma institución. Este cuestionario SAHLSA está compuesto por 50 ítems, que permiten dos opciones de respuesta (clave o distractor) más la posibilidad "no sé". Se aplica mediante un set de tarjetas que incluye el concepto general y las dos opciones de respuesta. El instrumento se aplica por un encuestador entrenado, que vierte los resultados en una planilla estándar.

Para la aplicación del cuestionario se capacitó a un grupo de estudiantes universitarios en base a las instrucciones sugeridas por los autores que diseñaron el test ${ }^{10}$. Para el análisis, primero se realizó un análisis factorial exploratorio para simplificar la compleja y multivariada estructura de los datos, formulando hipótesis acerca de esta, las que luego fueron puestas a prueba con el análisis factorial confirmatorio ${ }^{12}$. Finalmente, en base a Teoría de Respuesta al Ítem ("Item Response Theory" o "IRT") se formula un modelo de dos parámetros, el que permite obtener la dificultad (b) y discriminación (a) para cada ítem del cuestionario SAHLSA.

El "Análisis Exploratorio de Factores" tuvo por finalidad detectar la existencia de variables latentes ${ }^{12}$ que se presume constituyen la base de las variables observadas empíricamente, a través de las preguntas del cuestionario ${ }^{13}$. Con esto se busca reducir la redundancia entre las variables utilizando el menor número posible de factores ${ }^{12}$. Debido a que el test SAHLSA utiliza variables dicotómicas, se construyó la matriz de correlación del análisis factorial en base a correlaciones tetracóricas ${ }^{14}$.

El "Análisis Confirmatorio de Factores", permitió confirmar el número de factores latentes y la naturaleza de la estructura de estos en los datos $^{12}$, específicamente en el modelo formulado, la covarianza de los 50 ítems fue completamente explicada por la varianza del único factor sumada a la varianza de cada ítem en particular. Al aislar la varianza compartida por los 50 ítems, se pudo obtener una mejor medición del factor o variable latente, en este caso la "Alfabetización en Salud"15. Además se estimó el $\mathrm{R}^{2}$, que es análogo al entregado en una regresión lineal, pudiendo cuantificar el porcentaje de varianza de la variable observada que es explicada por el factor o ítem (en cual el indicador es cargado $)^{14}$.

Se utilizó como estimador WSLMV (Weighted Least Squares Mean Variance adjusted), pertinente para variables categóricas ${ }^{14}$. Se obtuvo diversos índices de bondad de ajuste del modelo planteado, entre estos: el Comparative fit index (CFI) que se relaciona con el grado de correlación presente entre los ítems del cuestionario; el Índice de ajuste Tucker-Lewis (TLI) que penaliza a medida que 
el modelo se complejiza ${ }^{16}$; el Root mean square error of approximation (RSMEA) que cuantifica la falta de ajuste del modelo especificado a la población ${ }^{14}$. Finalmente se evalúa estadísticamente el ajuste del modelo en relación al RSMEA $\left(\mathrm{H}_{0}\right.$ : RMSEA $\leq 0,05$ ).

Una limitación importante de la Teoría Clásica de los Test es que la habilidad de la persona para responder en una evaluación y la dificultad de los ítems, no se pueden determinar por separado. Además, se sabe que la precisión al estimar la confiabilidad y el error estándar varían según el nivel de habilidad, sin embargo, la teoría clásica entrega sólo una medida de ambos parámetros ${ }^{17}$. Por tal motivo, se utilizó la Teoría de Respuesta al Ítem ("Item Response Theory" o IRT) que es una familia de modelos estadísticos que relacionan rasgos latentes como una habilidad, en este caso la "Alfabetización en Salud", con la probabilidad de respuesta correcta de un ítem en una evaluación, siendo ampliamente utilizados en psicometría y recientemente en Salud ${ }^{18}$. En base a IRT se realizó un modelo de dos parámetros (2PL), el que permite obtener la dificultad (b) y discriminación (a) para cada ítem. El parámetro de dificultad corresponde al punto en la escala en que la habilidad medida equivale a una probabilidad de 0,5 de obtener una respuesta correcta; y el parámetro de discriminación estima si el ítem puede diferenciar entre los diferentes niveles de habilidad o en este caso "Alfabetización en Salud" de los encuestados.

Como punto de corte para el parámetro de discriminación se consideró entre 0,8 y 2,5, y para dificultad entre $-3 \mathrm{y}+3^{19}$. Finalmente, se obtuvieron las curvas características de cada ítem, las que permiten explorar en forma gráfica los parámetros mencionados. En estas, un ítem con mayor poder de discriminación presentará una mayor pendiente.

Se utilizó el programa MPLUS v7.3 y el programa $\mathrm{R}$, el primero para realizar el análisis exploratorio y confirmatorio de factores, mientras que el segundo para obtener las curvas características del ítem. Se consideró un nivel de significancia de $5 \%$ para los contrastes de hipótesis realizados.

\section{Resultados}

El análisis exploratorio arrojó varios factores con eigen value por sobre 1 , sin embargo, el factor con mayor valor es más de tres veces mayor que el siguiente. Lo anterior estaría dando cuenta que los ítems son indicadores de un único factor latente, en este caso la "Alfabetización en Salud". Dicho factor común explica $31,37 \%$ de la varianza de los puntajes del cuestionario y al ser retenido se constata que en su mayoría las cargas factoriales de cada pregunta están por sobre 0,4 (Tabla 1 ).

En cuanto a la bondad de ajuste del modelo planteado en el análisis confirmatorio, el índice CFI alcanzó un valor de 0,93 . Se ha señalado que un razonable punto de corte para considerar un buen ajuste sería $0,90^{14}$, el que en este caso resulta pertinente debido a que las correlaciones entre las variables no fueron altas. El índice TLI fue 0,927 , siendo adecuado ya que el punto de corte es 0,90 . Además, el valor del RSMEA fue 0,044 , encontrándose dentro del rango de ajuste preciso $(<0,05)^{20,21}$, ubicándose el límite superior del intervalo de confianza $(0,054)$ dentro de un ajuste considerado aceptable. Finalmente, el test "Close fit" no fue estadísticamente significativo $(\mathrm{p}=0,828)$ por lo no que es posible rechazar la hipótesis de un buen ajuste del modelo ${ }^{22}$.

El cuestionario posee adecuada validez de constructo, esto tomando en consideración los índices de bondad de ajuste y el $\mathrm{R}^{2}$ de cada uno de sus reactivos (Tabla 2). Pese a los buenos indicadores globales es necesario considerar la pertinencia de los ítems: "incesto", "ojo" y "herpes" debido a que su $\mathrm{R}^{2}$ denota la baja varianza explicada en sus respuestas por el factor latente "Alfabetización en Salud". Además, se evaluó la confiabilidad del instrumento mediante el coeficiente de confiabilidad de $\mathrm{K}$-Richarson, el que arrojó un buen resultado $(\mathrm{KR} 20=0,9255)$.

En el caso de utilizar Ítem Response Theory (IRT), con un punto de corte para el parámetro de discriminación entre 0,8 y 2,5 , y una dificultad entre $-3 y+3$, se deberá considerar la pertinencia de los ítems "próstata", "empleo", "incesto", "rectal”, "ojo", "Impétigo", "Comportamiento", "Sífilis", "Herpes", "riñón" "Potasio" y "Vesícula Biliar". Esto basándonos en los índices de dificultad y discriminación (Tabla 3).

En la Figura 1 se observan las curvas características del ítem tanto para los con menor y mayor dificultad, como para los más y menos discriminantes. 
Tabla 1. Carga factorial del análisis exploratorio de factores con correlaciones tetracóricas del cuestionario: "Short Assessment of Health Literacy for Spanish-speaking Adults"

\begin{tabular}{|c|c|}
\hline Ítem & Carga \\
\hline 1. Próstata & 0,344 \\
\hline 2. Empleo & 0,367 \\
\hline 3. Menstrual & 0,525 \\
\hline 4. Gripe & 0,774 \\
\hline 5. Avisar & 0,44 \\
\hline 6. Comidas & 0,697 \\
\hline 7. Alcoholismo & 0,892 \\
\hline 8. Grasa & 0,829 \\
\hline 9. Asma & 0,384 \\
\hline 10. Cafeína & 0,840 \\
\hline 11. Osteoporosis & 0,851 \\
\hline 12. Depresión & 0,307 \\
\hline 13. Estreñimiento & 0,632 \\
\hline 14. Embarazo & 0,791 \\
\hline 15. Incesto & $-0,091$ \\
\hline 16. Pastilla & 0,595 \\
\hline 17. Testículo & 0,659 \\
\hline 18. Rectal & 0,475 \\
\hline 19. Ojo & $-0,408$ \\
\hline 20. Irritación & 0,552 \\
\hline 21. Anormal & 0,635 \\
\hline 22. Estrés & 0,749 \\
\hline 23. Aborto espontáneo & 0,738 \\
\hline 24. Ictericia & 0,639 \\
\hline 25. Papanicolaou & 0,604 \\
\hline 26. Impétigo & 0,546 \\
\hline 27. Indicado & 0,594 \\
\hline 28. Ataque & 0,768 \\
\hline 29. Menopausia & 0,048 \\
\hline 30. Apéndice & 0,545 \\
\hline 31. Comportamiento & 0,231 \\
\hline 32. Nutrición & $-0,668$ \\
\hline 33. Diabetes & 0,701 \\
\hline 34. Sífilis & 0,314 \\
\hline 35. Inflamatorio & 0,723 \\
\hline 36. Hemorroides & 0,586 \\
\hline 37. Herpes & 0,348 \\
\hline 38. Alérgico & 0,330 \\
\hline 39. Riñón & 0,049 \\
\hline 40. Calorías & 0,583 \\
\hline 41. Medicamento & $-0,287$ \\
\hline 42. Anemia & 0,067 \\
\hline 43. Intestinos & 0,530 \\
\hline 44. Potasio & 0,522 \\
\hline 45. Colitis & 0,668 \\
\hline 46. Obesidad & 0,325 \\
\hline 47. Hepatitis & 0,746 \\
\hline 48. Vesícula Biliar & 0,354 \\
\hline 49. Convulsiones & 0,505 \\
\hline 50. Artritis & 0,258 \\
\hline
\end{tabular}

Tabla 2. $R^{2}$ de cada una de los ítems del cuestionario: "Short Assessment of Health Literacy for Spanish-speaking Adults"

\begin{tabular}{|c|c|c|}
\hline Ítem & $\mathbf{R}^{2}$ & P value \\
\hline 1. Próstata & 0,162 & 0,023 \\
\hline 2. Empleo & 0,278 & 0,006 \\
\hline 3. Menstrual & 0,602 & 0,000 \\
\hline 4. Gripe & 0,823 & 0,000 \\
\hline 5. Avisar & 0,451 & 0,001 \\
\hline 6. Comidas & 0,736 & 0,000 \\
\hline 7. Alcoholismo & 0,908 & 0,000 \\
\hline 8. Grasa & 0,870 & 0,000 \\
\hline 9. Asma & 0,588 & 0,000 \\
\hline 10. Cafeína & 0,873 & 0,000 \\
\hline 11. Osteoporosis & 0,949 & 0,000 \\
\hline 12. Depresión & 0,367 & 0,000 \\
\hline 13. Estreñimiento & 0,470 & 0,000 \\
\hline 14. Embarazo & 0,738 & 0,000 \\
\hline 15. Incesto & 0,004 & 0,731 \\
\hline 16. Pastilla & 0,712 & 0,000 \\
\hline 17. Testículo & 0,550 & 0,000 \\
\hline 18. Rectal & 0,154 & 0,012 \\
\hline 19. Ojo & 0,112 & 0,274 \\
\hline 20. Irritación & 0,421 & 0,000 \\
\hline 21. Anormal & 0,472 & 0,000 \\
\hline 22. Estrés & 0,814 & 0,000 \\
\hline 23. Aborto espontáneo & 0,753 & 0,000 \\
\hline 24. Ictericia & 0,487 & 0,000 \\
\hline 25. Papanicolaou & 0,494 & 0,000 \\
\hline 26. Impétigo & 0,247 & 0,001 \\
\hline 27. Indicado & 0,481 & 0,000 \\
\hline 28. Ataque & 0,628 & 0,000 \\
\hline 29. Menopausia & 0,470 & 0,000 \\
\hline 30. Apéndice & 0,697 & 0,000 \\
\hline 31. Comportamiento & 0,232 & 0,011 \\
\hline 32. Nutrición & 0,669 & 0,000 \\
\hline 33. Diabetes & 0,731 & 0,000 \\
\hline 34. Sífilis & 0,207 & 0,007 \\
\hline 35. Inflamatorio & 0,838 & 0,000 \\
\hline 36. Hemorroides & 0,762 & 0,000 \\
\hline 37. Herpes & 0,095 & 0,080 \\
\hline 38. Alérgico & 0,626 & 0,000 \\
\hline 39. Riñón & 0,354 & 0,006 \\
\hline 40. Calorías & 0,268 & 0,001 \\
\hline 41. Medicamento & 0,671 & 0,000 \\
\hline 42. Anemia & 0,677 & 0,000 \\
\hline 43. Intestinos & 0,680 & 0,000 \\
\hline 44. Potasio & 0,225 & 0,002 \\
\hline 45. Colitis & 0,512 & 0,000 \\
\hline 46. Obesidad & 0,578 & 0,000 \\
\hline 47. Hepatitis & 0,713 & 0,000 \\
\hline 48. Vesícula Biliar & 0,219 & 0,014 \\
\hline 49. Convulsiones & 0,526 & 0,000 \\
\hline 50. Artritis & 0,386 & 0,005 \\
\hline
\end{tabular}

En negrita se denotan los ítem con bajo y no significativo $R^{2}$ 
Validación de un test de para medir alfabetización en salud - M. J. Monsalves et al

Tabla 3. Dificultad y discriminación de los ítems del "Short Assessment of Health Literacy for Spanish-speaking Adults"

\begin{tabular}{|c|c|c|c|c|}
\hline Ítem & Dificultad & P value & Discriminación & P value \\
\hline 1. Próstata & $-1,50$ & 0,000 & 0,44 & 0,000 \\
\hline 2. Empleo & $-1,84$ & 0,000 & 0,62 & 0,000 \\
\hline 3. Menstrual & $-2,01$ & 0,000 & 1,23 & 0,001 \\
\hline 4. Gripe & $-1,44$ & 0,000 & 2,16 & 0,003 \\
\hline 5. Avisar & $-1,95$ & 0,000 & 0,91 & 0,000 \\
\hline 6. Comidas & $-1,38$ & 0,000 & 1,67 & 0,000 \\
\hline 7. Alcoholismo & $-1,30$ & 0,000 & 3,14 & 0,000 \\
\hline 8. Grasa & $-1,33$ & 0,000 & 2,59 & 0,000 \\
\hline 9. Asma & $-2,03$ & 0,000 & 1,20 & 0,000 \\
\hline 10. Cafeína & $-1,04$ & 0,000 & 2,63 & 0,001 \\
\hline 11. Osteoporosis & $-1,60$ & 0,000 & 4,32 & 0,116 \\
\hline 12. Depresión & $-1,95$ & 0,000 & 0,76 & 0,000 \\
\hline 13. Estreñimiento & $-1,56$ & 0,000 & 0,94 & 0,000 \\
\hline 14. Embarazo & $-1,45$ & 0,000 & 1,68 & 0,000 \\
\hline 15. Incesto & $-7,21$ & 0,459 & 0,06 & 0,493 \\
\hline 16. Pastilla & $-1,74$ & 0,000 & 1,57 & 0,000 \\
\hline 17. Testículo & $-1,01$ & 0,000 & 1,11 & 0,000 \\
\hline 18. Rectal & $-1,02$ & 0,003 & 0,48 & 0,000 \\
\hline 19. Ojo & $-4,66$ & 0,016 & 0,36 & 0,052 \\
\hline 20. Irritación & $-1,57$ & 0,000 & 0,85 & 0,000 \\
\hline 21. Anormal & $-1,56$ & 0,000 & 0,95 & 0,000 \\
\hline 22. Estrés & $-1,73$ & 0,000 & 2,09 & 0,024 \\
\hline 23. Aborto espontáneo & $-1,43$ & 0,000 & 1,75 & 0,000 \\
\hline 24. Ictericia & $-1,39$ & 0,000 & 0,98 & 0,000 \\
\hline 25. Papanicolaou & $-1,86$ & 0,000 & 0,99 & 0,000 \\
\hline 26. Impétigo & $-0,80$ & 0,003 & 0,57 & 0,000 \\
\hline 27. Indicado & $-0,97$ & 0,000 & 0,96 & 0,000 \\
\hline 28. Ataque & $-1,28$ & 0,000 & 1,30 & 0,000 \\
\hline 29. Menopausia & $-2,89$ & 0,000 & 0,94 & 0,000 \\
\hline 30. Apéndice & $-2,37$ & 0,000 & 1,52 & 0,000 \\
\hline 31. Comportamiento & $-2,45$ & 0,000 & 0,55 & 0,000 \\
\hline 32. Nutrición & $-2,76$ & 0,000 & 1,42 & 0,000 \\
\hline 33. Diabetes & $-1,71$ & 0,000 & 1,65 & 0,000 \\
\hline 34. Sífilis & $-1,93$ & 0,000 & 0,51 & 0,000 \\
\hline 35. Inflamatorio & $-1,70$ & 0,000 & 2,28 & 0,004 \\
\hline 36. Hemorroides & $-1,79$ & 0,000 & 1,79 & 0,013 \\
\hline 37. Herpes & $-1,50$ & 0,003 & 0,33 & 0,002 \\
\hline 38. Alérgico & $-2,28$ & 0,000 & 1,29 & 0,000 \\
\hline 39. Riñón & $-3,03$ & 0,000 & 0,74 & 0,000 \\
\hline 40. Calorías & $-0,90$ & 0,001 & 0,61 & 0,000 \\
\hline 41. Medicamento & $-2,76$ & 0,000 & 1,43 & 0,000 \\
\hline 42. Anemia & $-2,75$ & 0,000 & 1,45 & 0,000 \\
\hline 43. Intestinos & $-2,74$ & 0,000 & 1,46 & 0,000 \\
\hline 44. Potasio & $-0,77$ & 0,005 & 0,54 & 0,000 \\
\hline 45. Colitis & $-1,57$ & 0,000 & 1,03 & 0,000 \\
\hline 46. Obesidad & $-2,61$ & 0,000 & 1,17 & 0,001 \\
\hline 47. Hepatitis & $-1,55$ & 0,000 & 1,58 & 0,001 \\
\hline 48. Vesícula Biliar & $-1,97$ & 0,000 & 0,53 & 0,000 \\
\hline 49. Convulsiones & $-1,91$ & 0,000 & 1,05 & 0,000 \\
\hline 50. Artritis & $-2,51$ & 0,000 & 0,79 & 0,001 \\
\hline
\end{tabular}

En negrita se denotan los ítems cuya dificultad o discriminación se encuentra fuera del punto de corte establecido. 

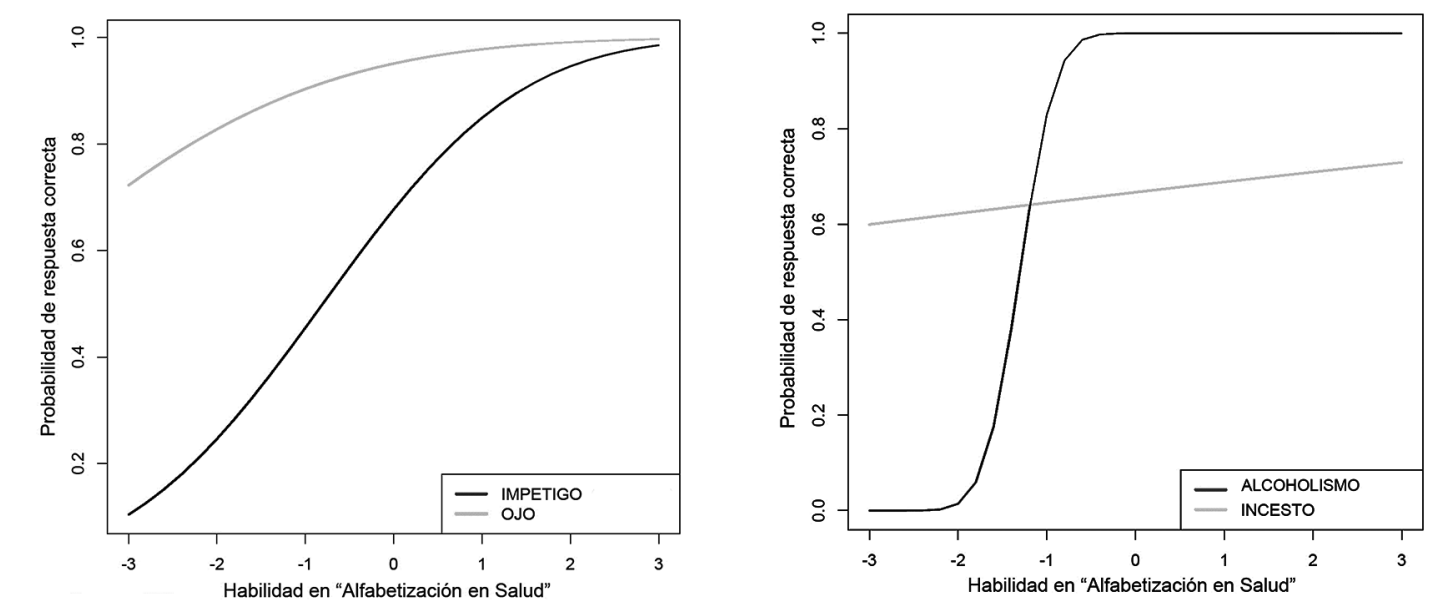

Figura 1. Curvas características del ítem para los con menor y mayor dificultad (izquierda) y discriminación (derecha) del cuestionario: "Short Assessment of Health Literacy for Spanish-speaking Adults".

\section{Discusión}

Este estudio de validación del Short Assessment of Health Literacy for Spanish-speaking Adults (SAHLSA-50) realizado en Chile permite proponer el test como una herramienta útil para evaluar alfabetización en salud en población adulta en el contexto nacional. El uso e incorporación de este cuestionario a la investigación local puede ser especialmente útil en las áreas de educación y promoción de la salud.

Al igual que lo referido por otros autores se observa que los ítems son indicadores de un único factor latente, que corresponde a "Alfabetización en salud", entendida como la capacidad de leer y entender los términos médicos comunes. Dicho factor común explica $31,37 \%$ de la varianza de los puntajes del cuestionario, siendo la mayoría de sus cargas factoriales por sobre $0,4^{10}$.

Se concluye que el cuestionario posee adecuada validez de constructo, esto tomando en consideración los índices de bondad de ajuste y el R2 de cada uno de sus reactivos. Sin embargo, a pesar de los buenos indicadores globales obtenidos es necesario poner atención en algunos ítems que estarían explicando poco de la "Alfabetización en salud" ("incesto", "ojo" y "herpes"), o que se encontraron fuera de los parámetros de dificultad y discriminación propuestos por los autores del test ("próstata", "empleo", “incesto", "rectal", "ojo", "Impétigo", "Comportamiento", "Sífilis", "Herpes", "riñón" "Potasio" y "Vesícula Biliar")" Estos ítems pueden ser incluso eliminados para la realización de estudios futuros con este test. No obstante, creemos que es necesario mantenerlos y evaluar la variación que estos pudiesen presentar en distintas poblaciones con variados contextos de nivel socioeconómico y educacional. Además, es recomendable realizar más estudios con este instrumento que permita considerar variables de caracterización individual que expliquen aún más la varianza observada.

Agradecimientos: Los autores agradecen al Dr. Shoou-Yih Lee de la Escuela de Salud Pública de la Universidad de Michigan, por permitir el uso y la validación del test Short Assessment of Health Literacy for Spanish-speaking Adults (SAHLSA-50) en Chile.

\section{Referencias}

1. Sørensen K, Van den Broucke S, Fullam J, Doyle G, Pelikan J, Slonska Z, et al. Health literacy and public health: a systematic review and integration of definitions and models. BMC Public Health 2012; 12 (1): 80.

2. Terry D, Wolf M. Health Literacy: Implications for Family Medicine. Fam Med 2004; 36 (8): 595-8. 
3. Peerson A, Saunders M. Health literacy revisited: what do we mean and why does it matter? Health Promot Int 2009; 24 (3): 285-96.

4. Davey J, Holden CA, Smith BJ. The correlates of chronic disease-related health literacy and its components among men: a systematic review. BMC Public Health 2015; 15 (1): 589.

5. Koh HK, Chan HTH, Kennedy H, Rudd RE, Chan HTH, Chan HTH. The Arc of Health Literacy. J Am Assoc 2015; 4: 7-8.

6. Huang Y-J, Wang Y-L, Wu T-Y, Chen C-T, Kuo KN, Chen S-S, et al. Validation of the short-form Health Literacy Scale in patients with stroke. Patient Educ Couns. Elsevier 2015; 98 (6): 762-70.

7. Machado ALG, Lima FET, Cavalcante TF, Araújo TL de, Vieira NFC. Instruments of health literacy used in nursing studies with hypertensive elderly. Rev Gaúcha Enferm. Universidade Federal do Rio Grande do Sul. Escola de Enfermagem 2014; 35 (4): 101-7.

8. Rodríguez SA, Roter DL, Castillo-Salgado C, Hooker GW, Erby LH. Translation and validation of a Spanish-language genetic health literacy screening tool. Health Psychol 2015; 34 (2): 120-9.

9. Toçi E, Burazeri G, Sørensen K, Kamberi H, Brand H. Concurrent validation of two key health literacy instruments in a South Eastern European population. Eur J Public Health 2015; 25 (3): 482-6.

10. Lee SD, Bender DE, Ruiz RE. Development of an Easyto-Use Spanish Health Literacy Test 2006; 1392-412.

11. Rencher A, Christensen M. Methods of Multivariate Analysis, 3rd Edition. New Jersey: Jhon Wiley \& Sons,
Inc; 2012.

12. Brown BL, Hendrix SB, Hedges DW, Smith TB. Multivariate analysis for the biobehavioral and social sciences. New Jersey: Jhon Wiley \& Sons, Inc; 2012.

13. Wang J, Wang X. Estructural Equation Modeling Aplications Using MPLUS. United Kingdom: Jhon Wiley \& Sons, Inc; 2012.

14. Acock AC. Discovering Structural Equation Modeling using Stata. 1st ed. College Station, Texas: StataCorp LP; 2013.

15. Bentler PM, Bonett DG. Significance test and goodness of fit in the analysis of covariance structures. Psychological Bulletin 1980; 88: 588-606.

16. Hays RD, Morales LS, Reise SP. Item response theory and health outcomes measurement in the 21st century. Med Care 2000; 38: 28-42.

17. Reise SP, Waller NG. Item response theory and clinical measurement. Annu Rev Clin Psychol 2009; 5: 27-48.

18. De Ayala, RJ. The Theory and Practice of Item Response Theory. New York, NY: Guilford Press 2009.

19. MacCallum RC, Browne MW, Sugawara HM. Power analysis and determination of simple size for covariance structure modeling. Pschological Methods 1996; 1: 13049.

20. Byrne BM. Structural Equation Modeling with LISREL, PRELIS, and SIMPLIS: Basic Concepts, Applications, and Programming. New Jersey: Lawrence Erlbaum Associates, Inc; 1998.

21. Byrne BM. Structural Equation Modeling with Mplus: Basic Concepts, Applications, and Programming. New York: Routledge, Taylor and Francis Group; 2012. 